\title{
The Association of Parametrial Invasion with the Expression of Vascular Endothelial Growth Factor-C and Other Factors in Squamous Cell Cervical Carcinoma Stage IB and IIA
}

\author{
Lilis $^{1, *}$, Nurjani Chairani Siregar ${ }^{2,3}$, Andrijono $^{4}$ \\ ${ }^{1}$ Department of Anatomical Pathology, School of Medicine and Health Science, Universitas Katolik Indonesia Atma Jaya, Jl. Pluit Raya No.2, \\ Jakarta, Indonesia \\ ${ }^{2}$ Department of Anatomical Pathology, Faculty of Medicine, Universitas Indonesia, Jl. Salemba Raya No.5, Jakarta, Indonesia \\ ${ }^{3}$ Eijkman Institute for Molecular Biology, Jl. Pangeran Diponegoro No.69, Jakarta, Indonesia \\ ${ }^{4}$ Department of Obstetrics and Gynecology, Faculty of Medicine, Universitas Indonesia, Jl. Salemba Raya No.5, Jakarta, Indonesia \\ *Corresponding author. E-mail:liliscsw@yahoo.co.id
}

Received date: May 13, 2019; Revised date: Jul 13, 2019; Accepted date: Jul 16, 2019

\section{Abstract}

$\mathrm{B}$ ACKGROUND: The presence of parametrial invasion in early stage squamous cell cervical cancer (SCCC) indeed indicates worse prognosis and need more adjuvant treatment. This study aimed to investigate the association between parametrial invasion and clinicohystopathology variables.

METHODS: This retrospective study used specimens of squamous cell carcinoma stage IB-IIA. The inclusion criteria were cervical cancer stage IB-IIA and post radical hysterectomy with lymph node dissection and exclusion criteria was damaged and or insufficient histological preparations. The clinicohystopathology variables included age, tumour size and stage, differentiation and inflammation grade, lymphatic vascular and parametrial invasion, and lymph node metastasis. Histopathology staining, immunohistochemistry examination, and vascular endothelial growth factor-C (VEGF-C) expression were

\section{Introduction}

The high incidence and mortality of the cervical cancer occur in developing countries. Approximately $85 \%$ of the prevalence and $88 \%$ of the deaths due to cervical cancer are found in those countries.(1) Mortality of the cervical cancer is also high in Indonesia. There were an estimated 9,498 deaths from cervical cancer annually, which was the evaluated according to the standard procedure. The independent-T, Chi square, and Fisher's exact test were applied to evaluate the association. The significance was set at $p<0.05$.

RESULTS: Seventy-five cases were eligible. Parametrial invasion was found in 15 cases $(20 \%)$. Three variables demonstrated a significant association with parametrial invasion, tumor size $>4 \mathrm{~cm}$ (OR 4.32, 95\% CI 1.29-14.38, $p=0.01$ ), lymphatic node metastasis (OR 3.90, 95\% CI $1.17-13.03, p=0.02$ ), and VEGF-C (OR $0.75,95 \%$ CI 0.65 $0.87, p=0.03$ ).

CONCLUSION: Tumor size of $>4 \mathrm{~cm}$ and lymph node metastasis (LNM) had a higher risk of parametrial invasion in SCCC stage IB-IIA.

KEYWORDS: cervical carcinoma, parametrial invasion, vascular invasion, peritumoral stroma, VEGF-C expression

Indones Biomed J. 2019; 11(3): 279-85 second leading cause of death among Indonesian women.(2) The highest mortality was related to cancer progression and poor prognosis as indicated by tumor invasion and lymph node metastasis (LNM). Parametrial tissue is the most frequent site of tumor cells dissemination. The presence of parametrial invasion indicates worse prognosis and more radical surgery is needed in early stage cervical cancer. $(3,4)$

The vascular endothelial growth factor C (VEGF-C) is a member of vascular endothelial growth factor (VEGF) 
family and has a role in angiogenesis. It is produced by tumor cells and peritumor. $(5,6)$ The VEGF-C plays an essential role in lymphangiogenesis predominantly via vascular endothelial growth factor receptor-3 (VEGFR-3).

(7) By promoting new lymph vessels, the VEGF-C can accelerate tumor cells metastasis to lymph nodes and distant organs.(8) Prior studies reported that increased VEGF-C was correlated with poor prognosis in several types of cancers. $(9,10)$ A prior study reported that VEGF-C was proven to be involved in lympangiogenesis in cervical cancer and its gene expression increase according to the increase in the clinical stage.(11)

Lymphatic changes due to lymphangiogenesis intratumoral and peritumoral have been believed to cause distinct biological effect on tumor behaviour and prognosis. However, the results vary between studies. Study in gastric cancer revealed that the intratumoral lymphatic vessel density (LVD) was more associated with the presence of LNM in early stage whereas peritumoral LVD was an independent risk factor for LNM and prognosis. $(12,13)$ Study on the expression of VEGF-C in peritumoral lymphatic has been performed. The results found increased expression of VEGF-C at the edge compared with the center of tumor were correlated with high peritumoral LVD, lymphovascular invasion (LVI), and nodal metastasis. These indicated that the peritumoral lympatic invasion was linked with tumor metastasis and expected to be an independent prognostic factor in cervical cancer.(14) On the contrary, study on gastric cancer reported that intratumoral, but not peritumoral, lymphatic vessel density (LVD) was closely related with LNM and poor prognosis.(15)

To the best of our knowledge, there may be fewer studies that investigate the association between parametrial invasion and other prognostic factors such as lymphatic invasion in peritumoral in cervical cancer. In addition, the relationship between parametrial invasion, VEGF-C expression, peritumoral lymph vessels with and LNM, and clinicopathological outcomes also have not been firmly established yet. The aim of the present study is to investigate the association of parametrial invasion with VEGF-C expression, LVI, LNM in cervical cancer stage IB-IIA.

\section{Methods}

This was a retrospective study. Tumor specimens were obtained from the Cipto Mangunkusumo Hospital, Jakarta and Hasan Sadikin Hospital, Bandung from September 2014 to January 2016. The inclusion criteria were set as follows: cervical cancer stage IB-IIA according to the 1998 Federation Internationale des Gynaecologistes et Obstetristes (FIGO) staging system and patients had undergone radical hysterectomy with lymph node dissection. Damaged and or insufficient amounts of paraffin blocks were excluded. The recent study was approved by the Research Ethic Committe of Faculty of Medicine, Universitas Indonesia (No. 564/ UN2.F1/ETIK/2014).

\section{Histopathologic Examination}

Histopathologic preparation was made with standard procedure. Initially, the tissues from specimens were fixed at $10 \%$ buffered formalin. The next sequential steps were paraffin-embedding and hematoxylin and eosin (H\&E) staining. Evaluations of H\&E slides of the cases were performed to confirm the diagnosis. The histopathologic features including histologic type, tumor staging and size, tumor differentiation, the presence of LVI, parametrium invasion, and LNM were assessed.

\section{Immunohistochemistry}

Deparaffinization of the 4- $\mu \mathrm{m}$-thick sections were performed in xylene, followed by rehydration in graded concentration of ethanol. The endogenous peroxidase activity within tissue was destructed by mixing with $3 \% \mathrm{H}_{2} \mathrm{O}_{2}$ in phosphate buffer solution (PBS) for 20 minutes. Antigen retrieval was attained by immersing the sections in $0.01 \mathrm{M}$ citrate buffer, pH 6.0 and heated in a decloaking chamber for 35 minutes. Nonspecific binding sites were blocked in phosphatebuffered saline (PBS) for 20 minutes at room temperature. Primary antibodies for VEGF-C (1:150, Abcam, Cambridge, UK) was used and the slides were incubated overnight at $4^{\circ} \mathrm{C}$. After rinsing twice with phosphate buffered saline (PBS) for $10 \mathrm{~min}$, the sections were then incubated with a secondary solution Starr trek Universal HRP Detection system ${ }^{\circledR}$ (Biocare, Birmingham, UK) for $30 \mathrm{~min}$ at room temperature. Ultimately, the 3,3'-diaminobenzidine (DAB) was used according to the manufacturer's instructions to visualize the peroxidase activity. Meyers hematoxylin was used to counterstain the sections. A positive staining was confirmed when brown color appeared. The sections were then cleaned using $\mathrm{dH}_{2} \mathrm{O}$. The positive control was a single slide with squamous cell cervical carcinoma (SCCC) showed strong whose the sections were stained with VEGF antibody. The negative control was achieved by omitting the primary antibody.

Cytoplasmic and membrane immunoreactivity in peritumor was evaluated semiquantitatively. The numbers of brown color stromal cell at peritumor area were evaluated 
under light microscopic Olympus CX21 (Olympus, Tokyo Japan) and digital camera Optilab (Optilab, Phoenix, USA) at five random high power field. The expression of VEGF-C was defined as 'low' if there was no staining of inflammatory cells or staining of less than 5\%, and defined as 'high' if the staining was more than $5 \%$. The labeling was evaluated by two pathologists independently. If the results were not in agreement, they had to discuss to reach an agreement.

\section{Statistical Analysis}

Numerical data were presented as mean \pm standard deviation (SD) whereas categorical data as frequency. Comparison between two groups was analyzed using an independent sample of T-test. The association between LNM and VEGF-C expression with clinicopathology features, was analyzed using Chi-Square or Fisher's exact test. The odds ratio (OR) and 95\% confidence interval (CI) were also determined. A $p$-value of $<0,05$ was considered significant. Statistical analysis was computed by using the Statistical Package for the Social Sciences (SPSS) software version 17.

\section{Results}

A total of seventy-five specimens were eligible. Table 1 presented the clinicopathologic variables. Most of the cases were tumor in stage IB with size of $\leq 4 \mathrm{~cm}$, well-moderate differentiated, and had well-moderate inflamation grade. Parametrial invasion and lymph nodes metastasis were found only in 15 (20\%) and 18 (24\%) patients, respectively. LVI was identified in half of the cases.

The VEGF-C showed heterogeneous immunohistochemical expression within the tumor. In peritumoral stroma VEGF-C showed moderate and strong staining intensity (Figure 1). VEGF-C expression in the peritumoral stroma was a predominant low expression (60 cases or $80 \%$ ), whereas only 15 (20\%) cases showed high VEGF-C expression. In 15 cases with high VEGF-C expression, only 1 case showed the presence of LNM and no cases of parametrial invasion.

Table 2 demonstrated the comparison between parametrial invasion and its association with the clinicopathological features. Age was not different between positive and negative parametrial invasion $(p=0.65)$. Parametrial invasion had association with tumor size, LNM, and VEGF-C expression. The OR for tumor size, LNM, expression of VEGF-C were 4.32 (95\% CI 1.29-14.38, $p=0.01$ ), 3.90 (95\% CI 1.17-13.03, $p=0.02), 0.75$ (95\% CI $0.65-0.87, p=0.03)$.
Table 1.Clinicohystopathology variables.

\begin{tabular}{|c|c|c|}
\hline \multirow{2}{*}{$\begin{array}{c}\begin{array}{c}\text { Clinicohistopathology } \\
\text { Variables }\end{array} \\
\text { Age (years, mean } \pm \text { SD) }\end{array}$} & \multicolumn{2}{|c|}{ Frequency (\%) } \\
\hline & & $46.2 \pm 7.9$ \\
\hline \multicolumn{3}{|l|}{ Stage } \\
\hline FIGO IB1-IB2 & 53 & $70.70 \%$ \\
\hline FIGO IIA1-IIA2 & 22 & $29.30 \%$ \\
\hline \multicolumn{3}{|l|}{ Tumour size } \\
\hline$\leq 4 \mathrm{~cm}$ & 46 & $61.30 \%$ \\
\hline$>4 \mathrm{~cm}$ & 29 & $38.70 \%$ \\
\hline \multicolumn{3}{|l|}{ Differentiation grade } \\
\hline Well-moderate & 58 & $77.30 \%$ \\
\hline Poor & 17 & $22.70 \%$ \\
\hline \multicolumn{3}{|l|}{ Inflammation grade } \\
\hline Mild-moderate & 43 & $57.30 \%$ \\
\hline Severe & 32 & $42.70 \%$ \\
\hline \multicolumn{3}{|l|}{ Lymphatic vascular invasion } \\
\hline Negative & 37 & $49.30 \%$ \\
\hline Positive & 38 & $50.70 \%$ \\
\hline \multicolumn{3}{|l|}{ Parametrial invasion } \\
\hline Negative & 60 & $80.00 \%$ \\
\hline Positive & 15 & $20.00 \%$ \\
\hline \multicolumn{3}{|l|}{ Lymph node metastasis } \\
\hline Negative & 57 & $76.00 \%$ \\
\hline Positive & 18 & $24.00 \%$ \\
\hline
\end{tabular}

The association between VEGF-C expression and clinicopathology variables was shown in Table 3. The results show there was no association between age, stage, tumor size, histological grade, degree of inflammation and LVI with VEGF-C expression. The same results were demonstrated with LNM variable, there was a tendency of decreased expression of VEGF-C in peritumoral cervical SCC with LNM compared without LNM $(p=0.09)$. A tendency between low expression of VEGF-C and LNM was noted, however, the association was not statistically significantly $(p=0.10)$. The expression of VEGF-C is only related with parametrial invasion (in Table 2, $p=0.03$ ). There was no relationship between VEGF-C expression and other clinicohystopathology factors.

\section{Discussion}

Parametrial invasion indicates a poor prognosis and has implications for the need for more radical surgery. 

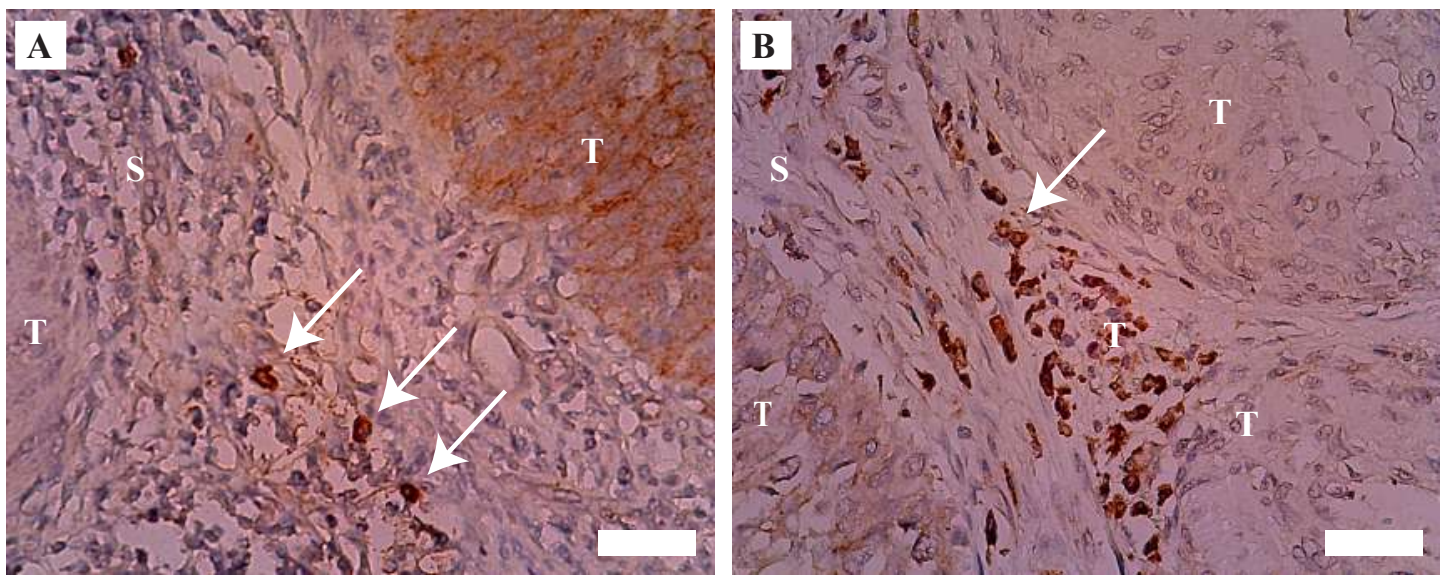

Figure 1. Expression of VEGF-C in peritumoral stroma cervical SCCC. A: low expression, less than 5\% inflammatory cells positive VEGF-C (arrow); B: high expression, more than 5\% inflammatory cells positive VEGF-C (arrow). T: tumor, S: stroma; white bar: $10 \mu \mathrm{m}$.

Parametrial tissue resection during radical hysterectomy, however may cause partial denervation of the autonomic nerve resulting in postoperative complications. The factors related to parametrial invasion in the early stage of SCCC should be identified for determining the risk of parametrial involvement. Our study found parametrial invasion occurred in $20 \%$ ( 15 cases). Parametrial invasion was related to tumor size of $>4 \mathrm{~cm}, \mathrm{LNM}$, and low expression of VEGF-C but not with other variables. Tumor size $>4 \mathrm{~cm}$, LNM, and high expression of VEGF-C had OR 4.32, 3.90 and 0.75, respectively. However, the association with VEGF-C was reversed and did not match with what was expected.

Table 2. The association between clinicopathology characteristics and parametrial invasion.

\begin{tabular}{|c|c|c|c|c|}
\hline \multirow{2}{*}{$\begin{array}{l}\text { Clinicopathological } \\
\text { Characteristics }\end{array}$} & \multicolumn{2}{|c|}{ Parametrial Invasion } & \multirow{2}{*}{$p$-value } & \multirow{2}{*}{ OR $(95 \% C I)$} \\
\hline & Positif & Negative & & \\
\hline Age (years) & $45.5 \pm 7.8$ & $46.5 \pm 8.0$ & 0.65 & \\
\hline \multicolumn{5}{|l|}{ Stage } \\
\hline FIGO IB1-IB2 & 11 & 42 & \multirow{2}{*}{0.99} & \multirow{2}{*}{$0.85(0.24-3.02)$} \\
\hline FIGO IIA1-IIA2 & 4 & 18 & & \\
\hline \multicolumn{5}{|l|}{ Tumour size $(\mathrm{cm})$} \\
\hline$\leq 4 \mathrm{~cm}$ & 5 & 41 & \multirow{2}{*}{0.01} & \multirow{2}{*}{$4.32(1.29-14.38)$} \\
\hline$>4 \mathrm{~cm}$ & 10 & 19 & & \\
\hline \multicolumn{5}{|l|}{ Differentiation grade } \\
\hline Well-moderate & 11 & 47 & \multirow{2}{*}{0.73} & \multirow{2}{*}{$1.32(0.36-4.82)$} \\
\hline Poor & 4 & 13 & & \\
\hline \multicolumn{5}{|l|}{ Inflammation grade } \\
\hline Mild-moderate & 2 & 13 & \multirow{2}{*}{0.72} & \multirow{2}{*}{$1.80(0.36-9.00)$} \\
\hline Severe & 13 & 47 & & \\
\hline \multicolumn{5}{|l|}{ Lymphatic vascular invasion } \\
\hline Negative & 5 & 32 & \multirow{2}{*}{0.17} & \multirow{2}{*}{$2.29(0.70-7.49)$} \\
\hline Positive & 10 & 28 & & \\
\hline \multicolumn{5}{|l|}{ Lymph node metastasis } \\
\hline Negative & 8 & 49 & \multirow{2}{*}{0.02} & \multirow{2}{*}{$3.90(1.17-13.03)$} \\
\hline Positive & 7 & 11 & & \\
\hline \multicolumn{5}{|l|}{ VEGF-C expression } \\
\hline Low & 15 & 45 & \multirow{2}{*}{0.03} & \multirow{2}{*}{$0.75(0.65-0.87)$} \\
\hline High & 0 & 15 & & \\
\hline
\end{tabular}


Table 3. The association between VEGF-C expression and clinicohystopathology variables.

\begin{tabular}{lccc}
\hline \multirow{2}{*}{ Clinicopathologic variables } & \multicolumn{2}{c}{ VEGF-C expression } & p-value \\
\cline { 2 - 3 } & Low (n=60) & High (15) & \\
\hline Stage & & & \\
$\quad$ FIGO IB1-IB2 & 45 & 8 & 0.12 \\
$\quad$ FIGO IIA1-IIA2 & 15 & 7 & \\
\hline Tumour size (cm) & 36 & 10 & 0.64 \\
$\leq 4$ cm & 24 & 5 & 1 \\
$>4$ cm & & & \\
\hline Histological grade & 46 & 12 & \\
Well differentiated & 14 & 3 & 0.42 \\
Moderate-poorly differentiated & & & \\
\hline Lymphatic vascular invasion & 31 & 6 & 0.09 \\
Negative & 29 & 9 & \\
Positive & & 14 & \\
\hline Lymph node metastasis & 43 & & \\
Negative & 17 & & \\
Positive & & & \\
\hline
\end{tabular}

In addition, VEGF-C has no association significantly with any variables.

Parametrium is the most frequent site of local spread of cervical cancer and its metastatic spread mainly lymphatic. (16) The tumor dissemination to parametrial tissue need resection, which is a more radical surgery causing known complications such as dysfunction of the bladder, erectile organ, and anorectal peristalsis.(17) Infertility is the most concerning complication for reproductive women who undergone radical surgery.(16) Previous studies have been performed to identify the risk factors for parametrial metastasis. This can help identify patients with low risk of parametrial metastasis and perform less radical surgery. Study on cervical carcinoma reported that patients with stage IB1 accompanied by superficial invasion $(<5 \mathrm{~mm})$ were at very low risk of parametrial invasion. Some previous studies demonstrate that parametrial invasion are related to tumor size, histologic grade, LNM, and LVI. The incidence of parametrial involvement is negative or low in primary tumor size of less than $2 \mathrm{~cm}$, no LVI, no LNM.(18-21) In agreement with some previous studies, this study reported any association between parametrial invasion with tumor size, LNM, and VEGF-C expression.

The association between parametrial invasion and VEGF-C expression was not evident in this study. With a significant $p$-value, the association was not as expected, in which parametrial invasion was more pronounced in low expression rather than in high expression of VEGF-C. The mechanism is not clearly understood, but it might be related to tumor cells invasion. Spreading tumor cells into lymphatic vessels could destroy the lymphatic endothelial lead to low expression of VEGF-C. However, this finding needs further evaluation to confirm whether VEGF-C indeed associate with parametrial invasion.

VEGF-C specifically acts on lymphatic endothelial cells through its recepto, VEGFR-3 to form new lymphatic vessels.(22,23) Study in cervical carcinoma indicated that VEGFR-3 expression was increased and correlated significantly with peritumoral LVD.(23) A similar results was reported which also found an increased VEGV-C expression was in accordance with clinical stage in SCCC. (11) Our study was not in agreement with those previous studies. The expression of VEGF-C had no association with LVI. We did not have not some definite explanation, but it might be related to histologic staining methods. With histologic appearance that resembles blood vessel, lymphatic vessel invasion could not be distinguished by the H\&E staining method.(24) To date, some more specific staining method (for example: D2-40, a monoclonal antibody) has been considered to have better capability to distinguish the vessels because it selectively stain lymphatic endothelial and exclusively found in the peritumoral area.(23,25-27) 
Both intra- or peritumoral lymphatic have a role on tumor cells dissemination and clinicopathology but the results vary between studies. It has been reported that peritumoral lymphatic vessels could be a potential prognostic marker for endometrial carcinoma.(28) A metaanalysis study reported that peritumoral lymphatic vessels was thought to be the main dissemination route in breast cancer.(29) On the other hand, a previous study found a conflicting result in which intratumoral LVD had a strong association with LNM and prognosis.(15) However, a more recent study reported that both intratumoral and peritumoral LVD correlate with LNM.(30) We examined peritumoral lymphatic as it was considered to be more often associated with nodal metastasis and clinicopathological features. In addition, intratumoral lymphatic is sometimes collapse because it has been invaded and destroyed by tumor cells. $(31,32)$ Our study did not find any correlations between LVI with LNM and VEGF-C. LVI has been regarded as a strong prognostic factor in breast cancer.(33,34) Yet, previous study evaluating the relationship between LVD, LVI, and LNM demonstrated that LVD correlated moderately and LVI correlated weakly with LNM.(29)

Not every tumor types demonstrates the association of VEGF-C with LNM. Research during recent years has provided more understanding towards the mechanism of local invasion and lymphatic spread. Nowadays, two lymphangiogenic growth factors, VEGF-C and VEGF-D, are recognized. They are produced by both tumor and stromal cells. These growth factors induce proliferation and migration of cancer cells through signaling via VEGFR-3 receptor found in lymphatic endothelial cells.(23) Secreted in an active form, VEGF-C and VEGF-D have an ability to bind VEGFR-3. The VEGF-C and VEGF-D will be processed by protease into an active form with higher affinity for VEGFR-3 in the extracellular environment. $(21,24)$ There was an indication of overlapping functions between these two growth factors, because a deficiency of VEGF-C can be replaced by VEGF-D.(24) Similar evidence was shown in colorectal cancer with decreased VEGF-D, in which the carcinoma may enhance the elevated levels of VEGF-C to bind with VEGFR-3 easily. The balance of different VEGF family members changes significantly, in which the ratio of VEGF-C and VEGF-D is essential for lymphatic invasion and metastasis.(22)

Limitations of this study have been documented. First, we did not examine LVD as an indicator for lymphatic invasion. Second, we did not use D2-40 staining even though it is a more specific staining for endothelial lymphatic vessels. The use of D2-40 could improve detection rates and avoid the false positive or negative as well. Third, VEGF-D expression was not examined. The VEFG-D, like VEGF-C, is also a potential regulator for lymphangiogenesis. The presence of VEGF-D expression is needed to confirm the lymphatic vascular invasion which could not be detected by VEGF-C.

\section{Conclusion}

In conclusion, this study indicates that parametrial invasion has association with tumor size and LNM, but not with VEGF-C expression. Tumor size of $>4 \mathrm{~cm}$ and positive LNM in stage IB-IIA squamous cell carcinoma increased the risk of parametrial invasion. Even with a significant result, the association of parametrial invasion and the VEGF-C is not conclusive because the direction of association is not appropriate. A further investigation is needed to confirm the association.

\section{Acknowledgements}

Authors would like to thanks laboratory staffs for technical support, and medical record officers for providing data.

\section{References}

1. Horton R. GBD 2010: understanding disease, injury, and risk. Lancet. 2012; 380: 2053-4

2. Viens LJ, Henley SJ, Watson M, Markowitz LE, Thomas CC, Thompson TD, et al. Human papillomavirus-associated cancersUnited States, 2008-2012. MMWR Morb Mortal Wkly Rep. 2016; 65: 661-6.

3. Chen Z, Huang K, Lu Z, Deng S, Xiong J, Huang J, et al. Risk model in stage IB1-IIB cervical cancer with positive node after radical hysterectomy. Onco Targets Ther. 2016; 9: 3171-9.

4. Wright JD, Grigsby PW, Brooks R, Powell MA, Gibb RK, Gao F, et al. Utility of parametrectomy for early stage cervical cancer treated with radical hysterectomy. Cancer. 2007; 110: 1281-6.

5. Kitadai Y, Amioka T, Haruma K, Tanaka S, Yoshihara M, Sumii K, et al. Clinicopathological significance of vascular endothelial growth factor (VEGF)-C in human esophageal squamous cell carcinomas. Int J Cancer. 2001; 93: 662-6.

6. Hadler-Olsen E, Wetting HL, Rikardsen O, Steigen SE, Kanapathippillai P, Grénman R, et al. Stromal impact on tumor growth and lymphangiogenesis in human carcinoma xenografts. Virchow Arch. 2010; 457: 677-92.

7. He Y, Rajantie I, Pajusola K, , Jeltsch M, Holopainen T, Yla-Herttuala $\mathrm{S}$, et al. Vascular endothelial cell growth factor receptor 3-mediated activation of lymphatic endothelium is crucial fortumor cells entry and spread via lymphatic vessels. Cancer Res. 2005; 65: 4739-46.

8. Lohela M, Bry M, Tammela T, Alitalo K. VEGFs and receptors involved in angiogenesis versus lymphangiogenesis. Curr Opin Cell Biol. 2009; 21: 154-65. 
9. Zhang Z, Luo G, Tang H, Cheng C, Wang P. Prognostic significance of high VEGF-C expression for patients with breast cancer: An update meta analysis. PLoSONE. 2016: 11: e0165725. doi: 10.1371/ journal.pone. 0165725 .

10. Liu P, Chen W, Zhu H, Liu B, Song S, Shen W, et al. Expression of VEGF-C correlates with a poor prognosis based on analysis of prognostic factors in 73 patients with esophageal squamous cell carcinomas. Jpn J Clin Oncol. 2009; 39: 644-50.

11. Franc M, Kachel-Flis A, Michalski B, Fila-Daniłow A, Mazurek U, Michalski M, et al. Lymphangiogenesis in cervical cancer evaluated by expression of the VEGF-C gene in clinical stage IB-IIIB. Prz Menopauzalmy. 2015; 14: 112-7.

12. Lee K, Park DJ, Choe G, Kim HH, Kim WH, Lee HS. Increased intratumoral lymphatic vessel density correlates with lymph node metastasis in early gastric carcinoma. Ann Surg Oncol. 2010; 17: 73-80.

13. Gao P, Zhou GY, Zhang QH, Xiang L, Zhang SL, Li C, et al. Clinicopathological significance of peritumoral lymphatic vessel density in gastric carcinoma. Cancer Lett. 2008; 263: 223-30.

14. Gombos Z, Xu X, Chu CS, Zhang PJ, Acs G. Peritumoral lymphatic vessel density and vascular endothelial growth factor-c expression in early-stage squamous cell carcinoma of the uterine cervix. Clin Cancer Res. 2005; 11: 8364-71.

15. Donizy P, Rudno-Rudzinska J, Halon A, Dziegala M, Kabarowski J, Frejlich E, et al. Intratumoral but not peritumoral Lymphatic Vessel Density measured by D2-40 expression predicts poor outcome in gastric cancer - ROC curve analysis to find cut-off point. Anticancer Research. 2014; 34: 3113-8.

16. Stegeman M, Louwen $M$, van der Velden J, ten Kate FJW, den Bakker MA, Burger CW, et al. The incidence of parametrial tumor involvement in select patients with early cervix cancer is too low to justify parametrectomy. Gynecol Oncol. 2007: 105; 475-80.

17. Lee SH, Cho KJ, Ko MH, Cho HY, Lee KB, Lim S. Factors associated with parametrial involvement in patients with stage IB1 cervical cancer: Who is suitable for less radical surgery? Obstet Gynecol Sci. 2018; 61: 88-94.

18. Kim MK, Kim JW, Kim MA, Kim HS, Chung HH, Park NH, et $a l$. Feasibility of less radical surgery for superficially invasive carcinoma of the cervix. Gynecol Oncol. 2010; 119: 187-191.

19. Frumovitz M, Sun CC, Schmeler KM, Deavers MT, dos Reis $\mathrm{R}$, Levenback $\mathrm{CF}$, et al. Parametrial involvement in radical hysterectomy specimens for women with early-stage cervical cancer. Obstet Gynecol. 2009; 114: 93-9.

20. Kamimori T, Sakamoto K, Fujiwara K, Umayahara K, Sugiyama Y, Utsugi $\mathrm{K}$, et al. Parametrial involvement in FIGO stage IB1 cervical carcinoma diagnostic impact of tumor diameter in preoperative magnetic resonance imaging. Int J Gynecol Cancer. 2011; 21: 34954.

21. Smith AL, Frumovitz M, Schmeler KM, dos Reis R, Nick AM, Coleman RL, et al. Conservative surgery in early-stage cervical cancer: what percentage of patients may be eligible for conization and lymphadenectomy? Gynecol Oncol. 2010; 119: 183-6.
22. Saharinen I, Tammela T, Karkkainen M, Alitalo K. Lymphatic vasculature: development, molecular regulation, and role in tumor metastasis and inflammation. Trends Immunol. 2004; 25: 387-95.

23. Botting SK, Fouad H, Elwell K, Rampy BA, Salama SA, Freeman $\mathrm{DH}$, et al. Prognostic significance of peritumoral lymphatic vessel density and vascular endothelial growth factor receptor 3 in invasive squamous cell cervical cancer. Translational Oncology. 2010; 3 : 170-5.

24. El-Gohary YM, Metwally G, Saad RS, Robinson MJ, Mesko T, Poppiti RJ. Prognostic significance of intratumoral and peritumoral lymphatic density and blood vessel density in invasive breast carcinomas. Am J Clin Pathol. 2008; 129: 578-86.

25. Wang XL, Fang JP, Tang RY, Chen XM. Different significance between intratumoral and peritumoral lymphatic vessel density in gastric cancer: a retrospective study of 123 cases. BMC Cancer. 2010; 10: 299. doi: 10.1186/1471-2407-10-299.

26. Schoppmann SF, Birner P, Studer P, Breiteneder-Geleff S. Lymphatic microvessel density and lymphovascular invasion assessed by antipodoplanin immunostaining in human breast cancer. Anticancer Res. 2001; 21: 2351-5.

27. Urabe A, Matsumoto T, Kimura M, Sonoue H, Kinoshita K. Grading system of lymphatic invasion according to D2-40 immunostaining is useful for the prediction of nodal metastasis in squamous cell carcinoma of the uterine cervix. Hystopathology. 2006; 49: 493-7.

28. Gao Y, Liu Z, Gao F, Meng XY. High density of peritumoral lymphatic vessels is a potential prognostic marker of endometrial carcinoma: a clinical immunohistochemical method study. BMC Cancer. 2010; 10: 131. doi: 10.1186/1471-2407-10-131.

29. Zhang S, Zhang D, Yi S, Gong M, Lu C, Cai Y, et al. The relationship of lympatic vessel density, lymphovascular invasion, and lymph node metastasis in breast cancer: a systematic review and metaanalysis. Oncotarget. 2017; 8: 2863-73.

30. Zhang S, Yi S, Zhang D, Gong M, Cai Y, Zou L. Intratumoral and peritumoral lymphatic vessel density both correlate with lymph node metastasis in breast cancer. Sci Rep. 2017; 7: 40364. doi:10.1038/ srep40364.

31. Ji RC. Lymphatic endothelial cells, tumor lymphangiogenesis, and metastasis: new insight into intratumoral and peritumoral lymphatics. Cancer Metastasis Rev. 2006; 25: 677-94.

32. Leu AJ, Berk DA, Lymboussaki A, Alitalo K, Jain RK. Absence of functional lymphatics within a murine sarcoma: a molecular and functional evaluation. Cancer Res. 2000; 60: 4324-7.

33. He KW, Sun JJ, Liu ZB, Zhuo PY, Ma QH, Liu ZY, et al. Prognostic significance of lymphatic vessel invasion diagnosed by D2-40 in Chinese invasive breast cancers. Medicine (Baltimore). 2017; 96: e8490. doi: 10.1097/MD.0000000000008490.

34. Norhisham NF, Chong CY, Safuan S. Peritumoral lymphatic vessel density and invasion detected with immunohistochemical marker D240 is strongly associated with distant metastasis in breast carcinoma. BMC Clin Pathol. 2017; 17: 2. doi: 10.1186/s12907017-0041-4. 